\title{
Familiarity effects on memory search and visual search
}

\author{
ROBB M. GILFORD and JAMES F. JUOLA \\ University of Kansas, Lawrence, Kansas 66045
}

\begin{abstract}
A group of 10 subjects participated in a memory search task and a visual search task in different sessions. The subjects searched for a given target letter in three-, four-, and five-letter words and pronounceable nonwords. There were no significant differences in either the reaction time (RT) data or the error rates between the two tasks. Mean RT increased linearly with the number of letters in the display or in the memory set. Word trials produced faster responses than nonwords by about $40 \mathrm{msec}$ in all conditions. Errors also increased with set size and occurred more often as misses on positive trials than as false alarms. The overall similarity of the results from memory search and visual search tasks suggests that the component processes involved are the same.
\end{abstract}

Recognition involves the comparison of an encoded stimulus with information stored in memory to determine if a match can be found between the two. Insights into the nature of this comparison process have been gained from search tasks involving a single target character and a search set of one or more characters. The subject's task is to produce a positive response if the target is included in the search set and a negative response otherwise. Sternberg (1966) has developed a memory search paradigm in which the search set is presented first and held in memory in some form until the target is presented. The visual search analog of this task was used by Atkinson, Holmgren, and Juola (1969), in which a single target letter was presented first followed by a horizontal array of letters. The data from these two experiments were very similar, with mean reaction time (RT) increasing linearly with set size in a parallel fashion for positive and negative responses.

A direct comparison of visual search and memory search in a within-subjects design was reported by Townsend and Roos (1973). They used three subjects who participated in 11 sessions of memory search followed by 11 sessions of visual search. The search sets in both tasks were strings of from one to five consonants. The results from the two tasks were very similar to each other and to data from comparable search experiments. Mean RT increased fairly linearly with set size, and the positive RT function was parallel to, but below, the negative function in both tasks.

Townsend and Roos (1973) argued that the comparison process involving the target item and the search set items could take place in either an auditory

This research was supported in part by funds from Biomedical Sciences Support Grant RR-07037 from the National Institutes of Health and National Science Foundation Grant No. BMS74-12801 to the second author. Requests for reprints should be addressed to James F. Juola, Department of Psychology, University of Kansas, Lawrence, Kansas 66045. or visual "form system." Both are presumed to be limited-capacity storage systems capable of handling echoic or iconic inputs from sensory processors as well as auditory or visual images derived from long-term memory. A limited-capacity translator can operate to transform information from either store to a form appropriate for the other when necessary. Within this outline, various models for search tasks were discussed by Townsend and Roos, and the model eventually favored by them was not so much demanded by the data as it was supported by plausibility arguments. In their model, the target in visual search is maintained in the visual store and compared with visual representations of the display items. In memory search, the memory set is maintained in the auditory store to be compared with the auditory translation of the target item. A further assumption, such as that the rate of comparison is the same in both stores, is necessary to account for the data.

The present study was designed to compare performance measures in visual search and memory search tasks, with the order of the tasks counterbalanced between subjects in an attempt to equate practice effects. Further, the types of materials differed from those used by Townsend and Roos (1973) by being structurally regular and, in sone cases, familiar. That is, instead of being random strings of consonants, the search sets included vowels and were composed to be consistent with rules of English orthography. Half of the sets were pronounceable meaningless letter strings, and half were common words. Whatever the effects of meaningfulness and familiarity of the search sets on RT and error data, it is unlikely that these effects should be the same in both tasks if one is based on auditory comparisons and the other on visual comparisons. If similar results are again obtained in the two tasks, parsimony at least should dictate that 
comparison processes are based on the same types of internal information codes in memory search and in visual search. On the other hand, any differences that are found between the results in the two tasks could indicate whether encoding, comparison, or decision processes are involved in these differences.

\section{METHOD}

The subjects were two female and eight male volunteers, 18 to 28 years of age; six received course credit, and four were uncompensated for participation.

The stimuli consisted of 90 common words and 90 nonwords. Each set was equally divided into strings of three, four, and five letters. The words were selected from the Kučera and Francis (1967) word lists such that word frequency was approximately equated across the three lengths (mean frequency in English was about 260 occurrence per million words). In addition, no letters were repeated within the words, and they all contained a single syllable. The nonwords were made by rearranging the letters of each word to produce pronounceable one-syllable anagrams. In $18 \%$ of the cases, a single letter in the word had to be changed in order to produce an acceptable nonword. All items were typed on white $6 \times 9$ in. cards using an IBM Selectric typewriter and lowercase Courier typeface. When placed into an Iconix tachistoscope, they appeared centrally located in the field of view, with the five-letter items subtending a visual angle of slightly less than $1 \mathrm{deg}$.

The subjects participated in two 75-min sessions on separate days. Five subjects were randomly assigned to the memory search task on the first session and to the visual search task on the second session, and these conditions were reversed for the other five subjects. Both sessions began with 30 practice trials on the assigned task using materials similar to those used on experimental trials. The subjects initiated each trial by pressing a footswitch when given a ready signal by the experimenter. In visual search sessions, a single target letter was displayed for $1,500 \mathrm{msec}$, and in memory search sessions, a three- to five-letter search set was presented for the same duration. At $1,500 \mathrm{msec}$, a patterned masking field of overlapping uppercase Xs and Os was presented. At 2,000 msec, the mask was replaced by a word or nonword display in visual search and by a single target letter in memory search. This display remained on until the subject responded, and RT was measured from onset of the final display until a positive or negative response button was pushed. The assignment of positive and negative responses to the right- and left-hand response buttons was counterbalanced across subjects.

Each subject saw all 180 experimental search sets in a different random order on each day. Further, the target letters differed from Session 1 to Session 2 such that all search sets were used equally often on positive and negative trials. Within each session, half of the trials were positive and half were negative. On positive trials, the target items were included about equally often in all serial positions within the search sets.

\section{RESULTS}

The mean RTs for correct responses were found for each subject for the cells of a 2 by 3 by 2 by 2 factorial design. The factors correspond to task (visual search or memory search), set size (three, four, or five letters), familiarity (words or nonwords), and response type (positive or negative). An analysis of variance showed no significant effect of task, $F(1,9)=4.39, p>.05$. The analysis did show that RT increased with search set size $[F(2,18)=205.31$, $\mathrm{p}<.001]$, RT was shorter on word trials than on nonword trials $[F(1,9)=61.24, p<.001]$, and RT was shorter on positive trials than on negative trials $[F(1,9)=16.69, p<.01]$. None of the interactions were significant. In addition, no reliable effects were found relating positive RT to the serial position of the target within the search set. Since the difference between positive and negative RTs was about $38 \mathrm{msec}$ in all conditions, the levels of this factor were collapsed for clarity of presentation of the RT data. Mean RT as a function of set size, familiarity, and task is presented in Figure 1.

The analysis of variance for the arcsinetransformed error data found two significant effects: (1) error rate increased with set size (percent errors
Figure 1. Mean reaction times as functions of familiarity (words vs. nonwords) and search set size for memory search (left panel) and visual search (right panel). The equations represent least-squares linear fits to the data.

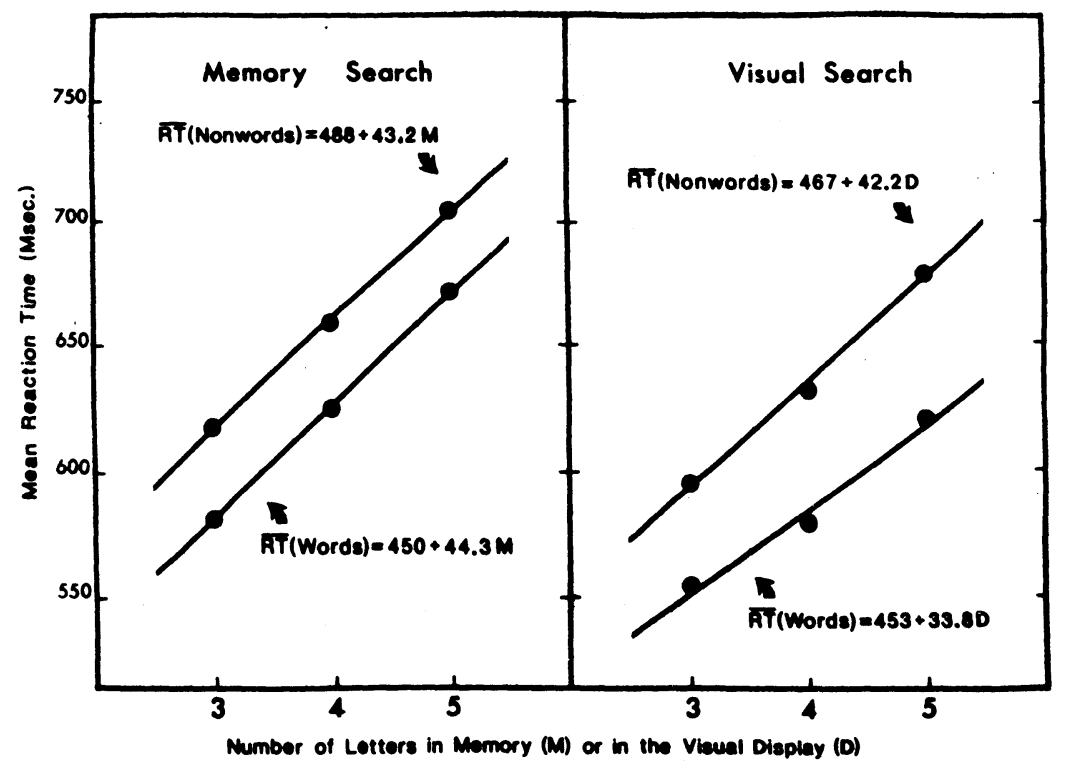


for Set Sizes 3, 4, and 5, respectively, were: 2.93, 3.52 , and 5.63), $F(2,18)=4.88, \mathrm{p}<.05$, and (2) more errors were made on positive trials $(5.33 \%)$ than on negative trials $(2.81 \%), F(1 ; 9)=8.05, \mathrm{p}<.05$. There were no other significant main effects or interactions in the error data.

\section{DISCUSSION}

The results generally replicated Townsend and Roos' (1973) findings of no significant differences in RT or error data between visual search and memory search tasks. Although mean RT, slopes of the RT by set size functions, and overall error rate were all less for visual search than for memory search, none of these differences reached statistical significance. The present study included search sets that differed with respect to meaningfulness and familiarity, and mean RT was less when the search sets were common words than when they were pronounceable nonwords. However, this effect did not interact with the type of search task. For these reasons, it appears that any theoretical account of the processes involved in visual search and memory search must take on much the same form for the two tasks.

Sternberg $(1969,1975)$ has done the most extensive theoretical work concerning processes involved in short-term memory search, and his exhaustive scanning model has proven its robustness in accounting for the results from numerous experiments. The general outline of the model would seem adequate to represent the processes involved in both tasks used in the present study. In Sternberg's model. it is first assumed that the memory search set (or the target item) is held in some form in memory while the subject awaits the onset of the target item (or the visual search set). The four stages involved in the model begin with an encoding process that operates on the target or the visual display to get it into a form that can be compared with the item or items held in memory. At this point, it is possible that either the information in memory or the information abstracted from the display could be transformed before processing could continue. Also, if the translator is limited in its processing capacity, the transformation time and thus overall RT would increase with set size if it is the search set that is transformed. Following encoding, an exhaustive scanning process is executed in which the target item is compared with each member of the search set. A binary decision is then made based on whether or not an acceptable match was found during the scan. In the final stage, the decision is mapped onto the appropriate response, which is then executed.

The simplest account of the present data would be to accept the Sternberg model at face value and assume that identical processes are involved in each of the corresponding stages of memory search and visual search. Thus the assumption would be that whatever the form of the information in memory (visual codes, auditory codes, or something more abstract than either), the target item or visual search set is encoded into this form, and then the comparison, decision, and response stages are executed in the same manner for both tasks. To account for the data, it would be necessary that the encoding stage be of approximately the same duration for a single letter as for a three- to five-letter item. This result would not be as surprising as might seem in light of Shiffrin and Gardner's (1972) demonstration of the lack of capacity limitations in the recognition of a small number of centrally presented letters. In addition, if translation is necessary before comparison can begin, then either the capacity of the translator is not exceeded by the types of materials used in the present study, or in both tasks it is either the search set or the target item that is translated.

A remaining problem is to explain the effect of familiarity or meaningfulness of the search set. Perhaps the only point that can be made with some certainty is that the comparison process was the same for words and nonwords. This conclusion is based on the additivity of familiarity and search set size, which indicates that familiarity affects some processing stage other than comparison. The exact locus of the effect cannot be determined from the present results, and it is not even certain that the same stage or stages are affected by familiarity in the two tasks. That is, the encoding stage could be facilitated in visual search by presenting familiar displays, but if familiarity is to affect encoding in a similar way in memory search, it is not obvious why a single target letter should be encoded faster when a word is held in memory rather than a nonword. Alternatively, it is possible that subsequent decision and response processes could be facilitated by a greater subjective confidence on word trials, but the lack of any error rate differences between word and nonword trials gives no support to this idea. Thus, it can only be stated that visual search and memory search are facilitated in quantitatively similar ways by varying the familiarity of the search sets, but this facilitation is not due to familiarity effects on the search process itself.

\section{REFERENCES}

Atrinson, R. C., Holmgren, J. E., \& Juola, J. F. Processing time as influenced by the number of elements in a visual display. Perception \& Psychophysics, 1969, 6, 321-327.

KuČERA, H., \& Francis, W. N. Computational analysis of present-day American English. Providence: Brown University Press, 1967.

ShIfFrin, R. M., \& Gardner, G. T. Visual processing capacity and attentional control. Journal of Experimental Psychology, 1972, 93, 72-82.

STERNBERG, S. High-speed scanning in human memory. Science, 1966, 153, 652-654.

STERnBERG, S. Memory scanning: Mental processes revealed by reaction-time experiments. American Scientist, 1969, 57. 421-457.

Sternberg, S. Memory scanning: New findings and current controversies. In D. Deutsch \& J. A. Deutsch (Eds.), Short-term memory. New York: Academic Press, 1975.

Townsend, J. T., \& Roos, R. N. Search time for single targets in multiletter stimuli with brief visual displays. Memory \& Cognition, 1973, 1, 319-332.

(Received for publication October 17, 1975.) 